\title{
SOLVING ELLIPTIC FINITE ELEMENT SYSTEMS IN NEAR-LINEAR TIME WITH SUPPORT PRECONDITIONERS
}

\author{
ERIK G. BOMAN*, BRUCE HENDRICKSON ${ }^{\dagger}$, AND STEPHEN VAVASIS ${ }^{\ddagger}$
}

\begin{abstract}
We show in this note how support preconditioners can be applied to a class of linear systems arising from use of the finite element method to solve linear elliptic problems. Our technique reduces the problem, which is symmetric and positive definite, to a symmetric positive definite diagonally dominant problem. Significant theory has already been developed for preconditioners in the diagonally dominant case. We show that the degradation in the quality of the preconditioner using our technique is only a small constant factor.
\end{abstract}

1. Introduction. Finite element discretizations of elliptic PDEs give rise to large sparse linear systems of equations. A topic of great interest is preconditioners for iterative solution of such systems. Our contribution in this paper has two parts: First, we show how SPD matrices of a particular form $K=A^{T} \bar{D}^{1 / 2} \bar{J}^{T} \bar{J} \bar{D}^{1 / 2} A$ can be well approximated by a symmetric diagonally dominant, M-matrix. Since significant theory has been developed for such matrices, we know they can be solved efficiently using preconditioned iterative methods. Second, we show that the stiffness matrix $K$ from a large class of finite elements for elliptic problems has the structure mentioned above.

The idea of approximating FEM systems by diagonally dominant matrices is not new; see for example [8]. In fact, our approach is similar to Gustafsson's in that we approximate each element matrix by a diagonally dominant matrix. In contrast to [8], we are able to rigorously prove bounds on the spectral properties of our approximation (and thus also the preconditioner). Our analysis uses the support theory framework described in [3] for analyzing condition numbers and (generalized) eigenvalues for preconditioned systems.

The support theory framework is briefly reviewed in Section 2, and then a new general-purpose result about support numbers is provided. In order to apply this result to finite element problems, a factorization of the finite element stiffness matrix $K$ in the form $K=A^{T} \bar{D}^{1 / 2} \bar{J}^{T} \bar{J} \bar{D}^{1 / 2} A$ is obtained, which is the topic of Sections 35. This factorization is found in two steps. First, we factor $K=A^{T} J^{T} D J A$ in a fairly natural fashion, i.e., in a fashion that has a direct derivation from finite element principles. Then we refactor $J^{T} D J$ as $\bar{D}^{1 / 2} \bar{J}^{T} \bar{J}^{1 / 2}$ in order to apply our new result. Analysis of the relevant condition numbers is provided in Section 6. Since our notation is somewhat formidable, we provide an explanatory example of how the method works for the commonly arising special case of piecewise linear finite elements in two dimensions in Section 7.

2. Support Theory. For $n \times n$ symmetric positive semidefinite (SPSD) matrices $A, B$ we define the support number to be

$$
\sigma(A, B)=\min \left\{t \in \mathbb{R} \mid \mathbf{x}^{T}(\tau B-A) \mathbf{x} \geq 0 \text { for all } \mathbf{x} \in \mathbb{R}^{n} \text { and for all } \tau \geq t\right\} .
$$

For historical reasons, the letter $\sigma$ is used for support numbers. Unfortunately, $\sigma$ is also commonly used to denote singular values. In this paper a $\sigma$ with one argument is a singular value, and with two arguments it is a support number.

\footnotetext{
*Sandia National Labs, Albuquerque, NM 87185-1111, egboman@sandia.gov.

†Sandia National Labs, Albuquerque, NM 87185-1111, bah@cs.sandia.gov.

${ }^{\ddagger}$ Department of Computer Science, 4130 Upson Hall, Cornell University, Ithaca, NY 14853, USA, vavasis@cs.cornell.edu.
} 
When $A$ and $B$ are symmetric positive definite (SPD) then $\sigma(A, B)=\lambda_{\max }(A, B)$, the largest generalized eigenvalue. When $B$ is a preconditioner for $A$, then the condition number of the preconditioned system is given by $\sigma(A, B) \sigma(B, A)$. Hence, by bounding the support numbers $\sigma(A, B)$ and $\sigma(B, A)$ we can bound the condition number. A number of algebraic techniques for bounding support numbers were given in [3]. For symmetric, diagonally dominant matrices, graph embedding techniques can also be used $[7,2,13]$. In the latter paper [13] it was shown that all symmetric, diagonally dominant systems can be solved in near-linear time. Our aim in this paper is to extend this result to FEM systems that are not diagonally dominant.

We will need some results from [3]. One is the triangle inequality,

$$
\sigma(K, M) \leq \sigma(K, \bar{K}) \sigma(\bar{K}, M),
$$

which shows that the the overall support number is bounded by the product of the support numbers in each step of the approximation $K \approx \bar{K} \approx M$.

A second result is the Symmetric-Product Support Theorem:

Theorem 2.1. Suppose $U \in \mathbb{R}^{n \times k}$ is in the range of $V \in \mathbb{R}^{n \times p}$. Then

$$
\sigma\left(U U^{T}, V V^{T}\right)=\min _{W}\|W\|_{2}^{2} \text { subject to } V W=U .
$$

We derive a couple corollaries from this theorem that will be useful in our analysis. We are interested in the case where $U=V G$ for some $G$.

Corollary 2.2. Let $V$ be as above and suppose $G$ is a matrix with $p$ rows. Then

$$
\sigma\left(V G G^{T} V^{T}, V V^{T}\right) \leq \sigma_{\max }(G)^{2},
$$

where $\sigma_{\max }$ denotes the largest singular value.

Proof. Let $U=V G$ and apply Theorem 2.1. This gives

$$
\sigma\left(V G G^{T} V^{T}, V V^{T}\right) \leq\|G\|_{2}^{2}=\sigma_{\max }(G)^{2} .
$$

Corollary 2.3. Let $V$ be as above and suppose $G$ is a rank-p matrix with $p$ rows. Then

$$
\sigma\left(V V^{T}, V G G^{T} V^{T}\right) \leq \sigma_{\min }(G)^{-2}
$$

where $\sigma_{\min }$ denotes the smallest singular value.

Proof. Let $G^{\dagger}$ denote the Moore-Penrose pseudoinverse of $G$. In the square case, $G^{\dagger}=G^{-1}$ since $G$ has full rank. Let $\hat{V}=V G$ and thus $\hat{V} G^{\dagger}=V$. Then

$$
\sigma\left(V V^{T}, V G G^{T} V^{T}\right)=\sigma\left(\hat{V} G^{\dagger} G^{\dagger^{T}} \hat{V}^{T}, \hat{V} \hat{V}^{T}\right)
$$

and the result follows from Corollary 2.2 and the fact that the singular values of $G^{\dagger}$ are the reciprocals of those of $G$. $\square$

We can now put these two corollaries together and get a result for the condition number. Define $\kappa(A, B)=\sigma(A, B) \sigma(B, A)$. When $A, B$ are SPD, then $\kappa(A, B)=$ $\kappa\left(B^{-1} A\right)$ where $\kappa$ denotes the standard spectral condition number.

TheOrem 2.4. Suppose $V$ is $n$ by $p$ and let $G$ be a matrix with $p$ rows and full row rank. Then

$$
\kappa\left(V G G^{T} V^{T}, V V^{T}\right) \leq \kappa(G)^{2} .
$$


Proof. The result follows from Corollary 2.2 and 2.3 and by using the fact $\kappa(G)=$ $\sigma_{\max }(G) / \sigma_{\min }(G)$.

The way we will apply this theorem is to let $V$ be a weighted vertex-edge incidence matrix. Then $V V^{T}$ is diagonally dominant while $V G G^{T} V^{T}$ is not (in general). Therefore, we now have a tool to approximate non-diagonally-dominant matrices.

3. The matrix approximation. Our main matrix factorization result is summarized by the following theorem whose various aspects and whose proof are explained in upcoming sections.

THEOREM 3.1. Suppose $K$ is the $n \times n$ assembled stiffness matrix of the standard order-p isoparametric finite element method applied to the elliptic boundary value problem given by (4.1) below. (The entries of this matrix are given by (4.7).) Let d denote the space dimension, $m$ the number of elements, $n$ the number of unconstrained nodes (i.e., nodes not on Dirichlet boundaries), and $q$ the number of Gauss points in the finite element quadrature scheme. Assume the quadrature scheme has positive weights and is exact for polynomials of degree $2 p-2$. Let $l$ denote the number of nodes per element. (For triangles in two dimensions, we have $l=(p+1)(p+2) / 2$. For tetrahedra in three dimensions, we have $l=(p+1)(p+2)(p+3) / 6$.) Then $K$ may be factored as $A^{T} J^{T} D J A$, where $A$ is an $(l-1) m \times n$ reduced node-arc incidence matrix, $J$ is a dqm $\times(l-1) m$ matrix that is well conditioned (see (6.6) and (6.7) for the bounds) and $D$ is a dqm $\times$ dqm positive definite diagonal matrix. Further, $J^{T} D J$ may be refactored as $\bar{D}^{1 / 2} \bar{J}^{T} \bar{J} \bar{D}^{1 / 2}$ where $\bar{D}$ is a $(l-1) m \times(l-1) m$ positive definite diagonal matrix and $\bar{J}$ is a dqm $\times(l-1) m$ matrix that is also well conditioned (see (6.9) and (6.10)).

This theorem can now be combined with Theorem 2.4 to obtain a good approximation $\bar{K}$ to the stiffness matrix $K$. In particular, we take $\bar{K}=A^{T} \bar{D} A$. Then in the context of Theorem 2.4, $V=A^{T} \bar{D}^{1 / 2}$ and $G=\bar{J}^{T}$. The theorem thus implies that the condition number of $\bar{K}$ with respect to $K$ depends only on the condition number of $\bar{J}$ for which we have good bounds. Then $\bar{K}$ can be preconditioned using techniques in the previous literature. The complete description is given below in Section 8 .

4. Finite element method. In this section, we explain the boundary value problem and the finite element method used to solve it as introduced by Theorem 3.1. The class of problems under consideration consists of finite-element discretizations of the following second-order elliptic boundary value problem. Find $u: \Omega \rightarrow \mathbb{R}$ satisfying

$$
\begin{aligned}
\nabla \cdot(\theta \nabla u) & =-f & & \text { on } \Omega, \\
u & =u_{0} & & \text { on } \Gamma_{1}, \\
\theta \partial u / \partial n & =g & & \text { on } \Gamma_{2} .
\end{aligned}
$$

Here, $\Omega$ is a bounded open subset of $\mathbb{R}^{d}$ (typically $d=2$ or $d=3$ ), $\Gamma_{1}$ and $\Gamma_{2}$ form a partition of $\partial \Omega, \theta$ is a given scalar field on $\Omega$ that is positive-valued everywhere and is sometimes called the conductivity, $f: \Omega \rightarrow \mathbb{R}$ is a given function called the forcing function, $u_{0}$ is a given function called the Dirichlet boundary condition and $g$ is another given function called the Neumann boundary condition.

For the rest of this section, we describe the isoparametric finite element method for solving (4.1). This material is quite standard and is covered by many monographs, e.g., [10]. Our reason for presenting it is to define our notation used in the next few sections.

We assume that $\Omega$ is discretized using a mesh $\mathcal{T}$ of $\Omega$, which is a finite set of elements $T \in \mathcal{T}$ that meet each other simplicially. Each $T \in \mathcal{T}$ is the image of a 
mapping function, that is, an orientation-preserving diffeomorphism $\phi_{T}: T_{0} \rightarrow T$, where $T_{0}$ is called the reference element. An assumption made for simplicity is that the reference element $T_{0}$ is the standard simplex, e.g., the triangle with vertices $(0,0)$, $(1,0),(0,1)$ when $d=2$ or the tetrahedron with vertices $(0,0,0),(1,0,0),(0,1,0)$, $(0,0,1)$ when $d=3$. Other common choices for reference elements would be a square in $2 \mathrm{D}$ or a cube in $3 \mathrm{D}$. We assume further that each $\phi_{T}$ is a $d$-variate polynomial of degree $p$ or less.

The $\phi_{T}$ 's are chosen so if $T, T^{\prime}$ are neighboring elements that have a common simplicial subface $F$, then $T_{0}$ has simplicial subfaces $F_{0}, F_{0}^{\prime}$ such that $\phi_{T}\left(F_{0}\right)=F$, $\phi_{T^{\prime}}\left(F_{0}^{\prime}\right)=F$, and there is an affine linear automorphism $\alpha$ of $T_{0}$ such that $\alpha\left(F_{0}\right)=F_{0}^{\prime}$ and such that $\left.\phi_{T}^{\prime} \circ \alpha\right|_{F_{0}}=\phi_{T}$. Here, $\left.\alpha\right|_{F_{0}}$ denotes the restriction of $\alpha$ to $F_{0}$. Usually, $\phi_{T}$ is chosen so that the element $T$ conforms well to the boundaries and satisfy certain regularity properties; see, e.g., [12]. Let the union of all $T \in \mathcal{T}$ be denoted $\tilde{\Omega}$. This set $\tilde{\Omega}$ is intended to be a close approximation to $\Omega$; exact agreement is not possible unless the boundary of $\Omega$ can be expressed as the union of images of polynomial maps of degree $p$ or less.

Let $V_{p, \mathcal{T}}$ denote the set of functions $\psi: \tilde{\Omega} \rightarrow \mathbb{R}$ such that (a) $\psi$ is continuous; (b) $\left.\psi\right|_{T}$ has the form $s \circ \phi_{T}^{-1}$, where $s$ is a scalar polynomial function of degree $p$ or less on the reference element $T_{0}$ and where $T$ is any member of $\mathcal{T}$; and (c) $\psi$ is identically zero on $\tilde{\Gamma}_{1}$. Here, $\tilde{\Gamma}_{1}$ is the approximation to $\Gamma_{1}$ that is an appropriately chosen subset of $\partial \tilde{\Omega}$.

Observe that $V_{p, \mathcal{T}}$ is a finite-dimensional vector space. Let $\bar{V}_{p, \mathcal{T}}$ be the same as $V_{p, \mathcal{T}}$ except that in place of condition (c), we require $\left.\left(\mathrm{c}^{\prime}\right) \psi\right|_{\Gamma_{1}}=\tilde{u}_{0}$ where $\tilde{u}_{0}$ is an approximation to the Dirichlet boundary condition $u_{0}$ on $\tilde{\Gamma}_{1}$. The exact definition of $\tilde{u}_{0}$ is immaterial for the results of this paper. Observe that $\bar{V}_{p, \mathcal{T}}$ is a coset of $V_{p, \mathcal{T}}$.

The isoparametric finite element method finds an approximate solution $\tilde{u}$ to (4.1) in the space $\bar{V}_{p, \mathcal{T}}$. For this purpose, a basis of $V_{p, \mathcal{T}}$ must be identified. The standard approach to defining a basis is as follows. In the reference element, position an evenly-spaced array of $l=(p+1)(p+2) / 2$ nodes when $d=2$ or of $l=(p+1)(p+$ $2)(p+3) / 6$ nodes when $d=3$. These nodes have coordinates of the form $(i / p, j / p)$ (in two dimensions) or $(i / p, j / p, k / p)$ (in three dimensions) where $i, j$ (and $k$ in $3 \mathrm{D}$ ) are nonnegative integers whose sum is at most $p$. Let these nodes be enumerated $z_{1}, \ldots, z_{l}$.

For each $T \in \mathcal{T}$, let $v_{T, 1}, \ldots, v_{T, l}$ denote $\phi_{T}\left(z_{1}\right), \ldots, \phi_{T}\left(z_{l}\right)$. These $l$ points are the nodes of $T$ listed with their local numbering. (A word about notation: we use ordinary italic type for scalars and for vectors of length $d$. We reserve bold type for long vectors, e.g., vectors of length $n$.) Because of the assumption of how neighboring $\phi_{T}$ 's are related, nodes that lie on a simplicial subface of $T$ exactly coincide with the corresponding subset of nodes of any other element $T^{\prime}$ that shares this simplicial subface. The global list of nodes (in which duplicates like this are counted only once) is denoted $w_{1}, \ldots, w_{n}, w_{n+1}, \ldots, w_{n^{\prime}}$. Here, the first $n$ nodes in this enumeration are in $\tilde{\Omega}-\tilde{\Gamma}_{1}$ while $w_{n+1}, \ldots, w_{n^{\prime}}$ are contained in $\tilde{\Gamma}_{1}$. There is an index mapping function, the local-to-global numbering map, carrying a pair $(T, i)$ where $T \in \mathcal{T}$ and $i \in\{1, \ldots, l\}$ to an index $j \in 1, \ldots, n^{\prime}$. Denote this map by $L G$.

Now, finally, we define a basis $\pi_{1}, \ldots, \pi_{n}$ for $V_{p, \mathcal{T}}$ as follows. We require $\pi_{i}\left(w_{j}\right)=$ $\delta_{i j}$ for $i=1, \ldots, n$ and $j=1, \ldots, n^{\prime}$, where $\delta$ is the Kronecker delta symbol. This nodal definition of $\pi_{i}$ is then interpolated over each element using $\phi_{T}$ 's to define a function on all of $\tilde{\Omega}$. In detail, on reference element $T_{0}$, let $N_{J}$ be the unique polynomial of degree $p$ or less such that $N_{J}\left(z_{I}\right)=\delta_{I J}$ for $I, J=1, \ldots, l$. This $N_{J}$ is 
called a shape function. Suppose $T$ contains global node $w_{j}$, and that $I$ is the index (between 1 and $l$ ) such that $L G(T, I)=j$. Then $\left.\pi_{i}\right|_{T}$ is defined to be $N_{I} \circ \phi_{T}^{-1}$. It is not hard to prove that the sequence $\pi_{1}, \ldots, \pi_{n}$ defined in this manner forms a basis of $V_{p, \mathcal{T}}$.

Now, returning to (4.1), we can derive its weak form by multiplying both sides of the PDE by a test function $t$, where $t$ is a suitably smooth scalar function on $\Omega$ such that $\left.t\right|_{\Gamma_{1}}=0$. Then integrate by parts on the left-hand side to obtain

$$
\int_{\Omega} \nabla t(x) \cdot \theta(x) \nabla u(x) d x=\int_{\Omega} t(x) f(x) d x+\int_{\Gamma_{2}} t(x) g(x) d x .
$$

The finite element method discretizes this weak form in the sense that it computes a $\tilde{u} \in \bar{V}_{p, \mathcal{T}}$ such that for all $t \in V_{p, \mathcal{T}}$

$$
\int_{\tilde{\Omega}} \nabla t(x) \cdot \theta(x) \nabla \tilde{u}(x) d x=\int_{\tilde{\Omega}} t(x) \tilde{f}(x) d x+\int_{\tilde{\Gamma}_{2}} t(x) \tilde{g}(x) d x
$$

where $\tilde{f}, \tilde{g}$ are approximations to $f$ and $g$. Since the weak form is linear in $t$, it suffices for the weak form to hold only for the basis of $V_{p, \mathcal{T}}$. Thus, we seek $\tilde{u} \in \bar{V}_{p, \mathcal{T}}$ such that for $i=1, \ldots, n$ :

$$
\int_{\tilde{\Omega}} \nabla \pi_{i}(x) \cdot \theta(x) \nabla \tilde{u}(x) d x=\int_{\tilde{\Omega}} \pi_{i}(x) \tilde{f}(x) d x+\int_{\tilde{\Gamma}_{2}} \pi_{i}(x) \tilde{g}(x) d x .
$$

The next observation is that since $\tilde{u} \in \bar{V}_{p, \mathcal{T}}$, it may be written in the form

$$
\tilde{u}=u_{B C}+\sum_{j=1}^{n} \xi_{j} \pi_{j},
$$

where $u_{B C}$ is a function in $\bar{V}_{p, \mathcal{T}}$ that is equal to $\tilde{u}_{0}$ on $\tilde{\Gamma}_{1}$, is zero at nodes $w_{1}, \ldots, w_{n}$ (i.e., all nodes not on $\tilde{\Gamma}_{1}$ ), and is interpolated in the standard way in between nodes, and $\xi_{1}, \ldots, \xi_{n}$ are initially unknown scalars. Because of the delta-property of the $\pi_{i}$ 's, we have $\tilde{u}\left(w_{i}\right)=\xi_{i}$ for all $i=1, \ldots, n$. Substituting (4.3) into (4.2) and rearranging reduces the computation of $\tilde{u}$ to the problem of solving

$$
K \boldsymbol{\xi}=\mathbf{f}
$$

where $\boldsymbol{\xi}$ is the vector $\left(\xi_{1}, \ldots, \xi_{n}\right)^{T}$, f is called the "load vector" and arises from contributions involving $\tilde{f}, \tilde{g}$ and $u_{B C}$, and $K$ is the $n \times n$ matrix whose entries are defined by

$$
K(i, j)=\int_{\tilde{\Omega}} \nabla \pi_{i}(x) \cdot \theta(x) \nabla \pi_{j}(x) d x .
$$

This matrix $K$ is called the assembled stiffness matrix of the problem. It is sparse, symmetric and positive definite. Symmetry is obvious; positive definiteness follows from a fairly straightforward argument that we omit, and sparsity follows because $K(i, j)$ is nonzero only if there is an element $T$ that contains both global nodes $w_{i}$ and $w_{j}$. (The proof of positive definiteness assumes that $\tilde{\Gamma}_{1}$ contains at least one node per connected component of $\tilde{\Omega}$. We make this assumption in our setting, although it is not required for our method.) 
Integral (4.4) is difficult to compute directly because evaluating $\pi_{i}$ requires evaluation of $\phi_{T}^{-1}$, which is a nontrivial matter. Fortunately, this difficulty is avoided by carrying out the integral over the reference domain following a change of variables as follows:

$$
\begin{aligned}
K(i, j) & =\sum_{T \in \mathcal{T}} \int_{T} \nabla \pi_{i}(x) \cdot \theta(x) \nabla \pi_{j}(x) d x \\
& =\sum_{T \in \mathcal{T}} \int_{T_{0}} \nabla_{x} \pi_{i}\left(\phi_{T}(z)\right) \cdot \theta\left(\phi_{T}(Z)\right) \nabla_{x} \pi_{j}\left(\phi_{T}(z)\right) \operatorname{det}\left(D \phi_{T}(z)\right) d z
\end{aligned}
$$

where the notation $\nabla_{x}$ means derivative with respect to the coordinates of $T$ (as opposed to derivative with respect to $z$, the coordinates of $T_{0}$ ). Here, $D$ denotes differentiation, hence $D \phi_{T}(z)$ is a $d \times d$ matrix. The integrand of (4.5) is evaluated using the chain rule for derivatives. Assume $w_{i}$ is a node of $T$ (else the above integral is 0 ). Let $I$ be the index such that $L G(T, I)=i$, so that $\left.\pi_{i}\right|_{T}=N_{I} \circ \phi_{T}^{-1}$. Then

$$
\begin{aligned}
\nabla_{x} \pi_{i}\left(\phi_{T}(z)\right) & =\nabla_{x} N_{I}(z) \\
& =D \phi_{T}(z)^{-1} \cdot \nabla_{z} N_{I}(z),
\end{aligned}
$$

and similarly for $\pi_{J}$. Here, $D \phi_{T}(z)^{-1}$ denotes the inverse of a $d \times d$ matrix (which exists since $\phi_{T}$ is assumed to be diffeomorphic). The ' ' notation in the previous formula indicates matrix-vector multiplication.

We assume that the entries of $K$ are not computed exactly but are obtained by a quadrature rule that we now discuss. Let $r_{1}, \ldots, r_{q}$ be points in the interior of the reference element $T_{0}$ called the Gauss points. (As is common practice, we use this terminology even if the quadrature rule is not related to Gaussian quadrature.) Let $\omega_{1}, \ldots, \omega_{q}$ be corresponding Gauss weights. We denote this quadrature rule (i.e., the set of ordered pairs $\left.\left(r_{1}, \omega_{1}\right), \ldots,\left(r_{q}, \omega_{q}\right)\right)$ by the symbol $\mathcal{Q}$. Then in place of $(4.5)$ we take

$$
K(i, j)=\sum_{T \in \mathcal{T}} \sum_{\nu=1}^{q} \nabla_{x} \pi_{i}\left(\phi_{T}\left(r_{\nu}\right)\right) \cdot \theta\left(\phi_{T}\left(r_{\nu}\right)\right) \nabla_{x} \pi_{j}\left(\phi_{T}\left(r_{\nu}\right)\right) \operatorname{det}\left(D \phi_{T}\left(r_{\nu}\right)\right) \omega_{\nu},
$$

in which $\nabla_{x} \pi_{i}\left(\phi_{T}\left(r_{\nu}\right)\right)$ is evaluated by substituting $z=r_{\nu}$ into the right-hand side of (4.6) and similarly for $\nabla_{x} \pi_{j}\left(\phi_{T}\left(r_{\nu}\right)\right)$.

5. Reformulation of the method. In this section we present a reformulation of the finite element method that leads to the factorization of $K$ on which the preconditioner is based. This factorization is related to one proposed in [15]. Let $\boldsymbol{\xi}$ be an arbitary vector in $\mathbb{R}^{n}$, and let $u \in V_{p, \mathcal{T}}$ be defined by

$$
u=\sum_{i=1}^{n} \xi_{i} \pi_{i}
$$

For an element $T$, let

$$
U_{T}=\sum_{I=1}^{l} \xi_{L G(T, I)} N_{I}
$$

With these definitions, it is clear that $\left.u\right|_{T} \circ \phi_{T}=U_{T}$. 
The assembled stiffness matrix is defined by (4.7) so that

$$
\begin{aligned}
\boldsymbol{\xi}^{T} K \boldsymbol{\xi} & =\sum_{i=1}^{n} \sum_{j=1}^{n} \xi_{i} \xi_{j} K(i, j) \\
& =\sum_{i=1}^{n} \sum_{j=1}^{n} \xi_{i} \xi_{j} \sum_{T \in \mathcal{T}} \sum_{\nu=1}^{q} \nabla_{x} \pi_{i}\left(\phi_{T}\left(r_{\nu}\right)\right) \cdot \theta\left(\phi_{T}\left(r_{\nu}\right)\right) \nabla_{x} \pi_{j}\left(\phi_{T}\left(r_{\nu}\right)\right) \operatorname{det}\left(D \phi_{T}\left(r_{\nu}\right)\right) \omega_{\nu} \\
& =\sum_{T \in \mathcal{T}} \sum_{\nu=1}^{q} \sum_{i=1}^{n} \xi_{i} \nabla_{x} \pi_{i}\left(\phi_{T}\left(r_{\nu}\right)\right) \cdot \theta\left(\phi_{T}\left(r_{\nu}\right)\right) \sum_{j=1}^{n} \xi_{j} \nabla_{x} \pi_{j}\left(\phi_{T}\left(r_{\nu}\right)\right) \operatorname{det}\left(D \phi_{T}\left(r_{\nu}\right)\right) \omega_{\nu} \\
& =\sum_{T \in \mathcal{T}} \sum_{\nu=1}^{q} \nabla_{x} u\left(\phi_{T}\left(r_{\nu}\right)\right) \cdot \theta\left(\phi_{T}\left(r_{\nu}\right)\right) \nabla_{x} u\left(\phi_{T}\left(r_{\nu}\right)\right) \operatorname{det}\left(D \phi_{T}\left(r_{\nu}\right)\right) \omega_{\nu} \\
& =\sum_{T \in \mathcal{T}} \sum_{\nu=1}^{q} \nabla_{x} U_{T}\left(r_{\nu}\right) \cdot \theta\left(\phi_{T}\left(r_{\nu}\right)\right) \nabla_{x} U_{T}\left(r_{\nu}\right) \operatorname{det}\left(D \phi_{T}\left(r_{\nu}\right)\right) \omega_{\nu} \\
& =\sum_{T \in \mathcal{T}} \sum_{i=1}^{q}\left(D \phi_{T}\left(r_{\nu}\right)^{-1} \nabla_{z} U_{T}\left(r_{\nu}\right)\right) \cdot \theta\left(\phi_{T}\left(r_{\nu}\right)\right) \\
& =\mathbf{v}^{T} D \mathbf{v} .
\end{aligned}
$$

In (5.3), we introduced a $d q m \times d q m$ diagonal matrix $D$ and a $d q m$-vector $\mathbf{v}$ with entries defined as follows. First, $m=|\mathcal{T}|$, the number of elements. The rows and columns of $D$ as well as the entries of $\mathbf{v}$ are indexed by ordered triples $(T, \nu, e)$ where $T \in \mathcal{T}, \nu \in\{1, \ldots, q\}$, and $e \in\{1, \ldots, d\}$. The $((T, \nu, e),(T, \nu, e))$ diagonal entry of $D$ is taken to be $\theta\left(\phi_{T}\left(r_{\nu}\right)\right) \operatorname{det}\left(D \phi_{T}\left(r_{\nu}\right)\right) \omega_{\nu} \alpha_{T}^{2}$ (independent of $e$ ). Here $\alpha_{T}$ is a positive scalar defined by (6.4) below. Because we want $D$ to be positive definite, we must impose the assumption mentioned in Theorem 3.1 that for all $\nu=1, \ldots, q, \omega_{\nu}>0$. There is some loss of generality with this assumption because a few popular finite element quadrature schemes (but certainly not all) use negative weights [6].

Entries of $\mathbf{v}$ are defined as follows: the $(T, \nu)$ block-entry of $\mathbf{v}$, which is a $d$ vector, is $D\left(\phi_{T}\left(r_{\nu}\right)\right)^{-1} \cdot \nabla U_{T}\left(r_{\nu}\right) / \alpha_{T}$. With these definitions of $D$ and $\mathbf{v}$, it is clear that $\mathbf{v}^{T} D \mathbf{v}$ is equal to the expression in (5.2).

Next, we write $\mathbf{v}=R S A \boldsymbol{\xi}$, where $R, S$ and $A$ are matrices defined as follows. First, $A$ is an $(l-1) m \times n$ matrix whose entries are all 0's, 1's and -1 's chosen according to the following pattern. For each element $T \in \mathcal{T}, A$ has a consecutive block of $l-1$ consecutive rows; let the rows of $A$ be indexed by $(T, j)$ for $j=2, \ldots, l$. The columns are indexed $1, \ldots, n$ in correspondence with global nodes $w_{1}, \ldots, w_{n}$. Row $(T, j)$ has a ' 1 ' in the column indexed by $L G(T, j)$ and a ' -1 ' in the column indexed by $L G(T, 1)$. Thus, most rows of $A$ have exactly two nonzero entries. In the case that $L G(T, j)>n$ (i.e., node $v_{T, j}$ lies in $\tilde{\Gamma}_{1}$ ), the ' 1 ' entry is omitted. Similarly, if $L G(T, 1)>n$, then the ' -1 ' entry is omitted. For this reason, a few rows of $A$ have just one nonzero entry or none at all.

Thus, $A$ is a reduced node-arc adjacency matrix of a graph defined on the nodes of $\mathcal{T}$. Each element gives rise to $l-1$ arcs in the graph. In particular, for each element $T \in T$, there is an arc joining each of its nodes $2, \ldots, l$ to node 1 . Note that the nodes of $\tilde{\Gamma}_{1}$ are all collapsed into a single supernode, and then the column corresponding to this supernode is deleted. (In the case that $\Omega$ has multiple connected components, the $\tilde{\Gamma}_{1}$ nodes of each component are distinct supernodes.) 
The product $A \boldsymbol{\xi}$ yields a vector with $(l-1) m$ entries. It is composed of $m$ blocks of $l-1$ entries. The block corresponding to $T \in \mathcal{T}$, which we denote by $\Delta \boldsymbol{\xi}_{T}$, is an $(l-1)$-vector of finite differences of entries of $\boldsymbol{\xi}$ (i.e., entries of the form $\xi_{i}-\xi_{j}$ ) that are associated with the $l$ nodes of $T$.

Next, let $S_{\mathcal{Q}, p}$ denote the $d q \times(l-1)$ matrix that carries out the following operation. Given an $(l-1)$-vector $\Delta \boldsymbol{\xi}_{T}$ that represents finite differences of nodal values of $u$ assigned to the nodes of $T_{0}$, we let $S_{\mathcal{Q}, p}$ be the matrix such that $S_{\mathcal{Q}, p} \Delta \boldsymbol{\xi}_{T}$ is the concatenation of $\nabla U_{T}\left(r_{1}\right), \ldots, \nabla U_{T}\left(r_{q}\right)$ where $U_{T}$ was defined by (5.1). (Because we have only finite differences of $\xi_{i}$ 's, $U_{T}$ cannot be recovered exactly from $\Delta \boldsymbol{\xi}_{T}$; rather, $U_{T}$ can be recovered only up to a constant additive term. This does not create a problem since $S_{\mathcal{Q}, p}$ is defined to produce the gradient of $U_{T}$ only, which does not require knowledge of this missing term.) This matrix $S_{\mathcal{Q}, p}$ depends on $p$ and $\mathcal{Q}$ but on no other aspect of the problem.

We now impose the following additional assumption mentioned in Theorem 3.1: the quadrature rule $\mathcal{Q}$ is exact for polynomials of degree $2 p-2$, i.e., if $\psi: T_{0} \rightarrow \mathbb{R}$ is a polynomial of degree $2 p-2$ or less, then

$$
\sum_{\nu=1}^{q} \psi\left(r_{\nu}\right) \omega_{\nu}=\int_{T_{0}} \psi(z) d z
$$

This assumption is quite reasonable since it is usually required anyway for accurate solution by finite element analysis: one wants accurate quadrature of $\nabla \pi_{i} \cdot \nabla \pi_{j}$. With this assumption in hand, we now have the following lemma.

Lemma 5.1. Assuming $\mathcal{Q}$ is exact for polynomials of degree $2 p-2$, matrix $S_{\mathcal{Q}, p}$ has rank $l-1$.

Proof. Let $\Delta \boldsymbol{\xi}_{T}$ be a vector of finite differences such that $S_{\mathcal{Q}, p} \Delta \boldsymbol{\xi}_{T}=0$. This means that the polynomial $\psi$ of degree at most $p$ that interpolates the underlying vector of $\xi_{i}$ 's at the nodes of $T_{0}$ has an identically zero gradient at all the Gauss points. Consider the polynomial $s=\nabla \psi \cdot \nabla \psi$. This polynomial has degree at most $2 p-2$ and is also identically zero at the Gauss points. Since the quadrature rule is exact for polynomials of this degree, $\int_{T_{0}} s(z) d z$ must be zero. But since $s$ is a nonnegative polynomial, this means $s$ must be identically zero, i.e., $\nabla \psi$ is identically zero, i.e., $\psi$ is a constant function. But in this case, $\Delta \boldsymbol{\xi}_{T}$ must be all zeros since its entries consist of finite differences of nodal values of $\psi$. Thus, we have shown that the only solution to $S_{\mathcal{Q}, p} \Delta \boldsymbol{\xi}_{T}=0$ is $\Delta \boldsymbol{\xi}_{T}=0$, which proves the theorem. $\mathrm{Z}$

Let us define $S$ to be the $d q m \times(l-1) m$ block-diagonal matrix consisting of $m$ copies of $S_{\mathcal{Q}, p}$ on the diagonal. In other words, the $((k-1) d q+1: k d q,(k-1)(l-1)+1$ : $k(l-1))$ submatrix of $S$ is a copy of $S_{\mathcal{Q}, p}$ for $k=1, \ldots, m$, and all other entries of $S$ are zeros. Then $S A \boldsymbol{\xi}$ is a vector whose entries are indexed by $(T, \nu, e)$ for $T \in \mathcal{T}$, $\nu \in 1, \ldots, q$ and $e \in 1, \ldots, d$. The $(T, \nu)$ block of entries of $S A \xi$ is a $d$-vector containing $\nabla U_{T}\left(r_{\nu}\right)$.

Recall that the $(T, \nu)$ block of entries of $\mathbf{v}$ contains $D \phi_{T}\left(r_{\nu}\right)^{-1} \nabla U_{T}\left(r_{\nu}\right) / \alpha_{T}$. Thus, to have the identity $\mathbf{v}=R S A \boldsymbol{\xi}$, we need to define a $d q m \times d q m$ block-diagonal matrix $R$ composed of $q m$ blocks of size $d \times d$ each, where the diagonal block indexed by $(T, \nu)$ is $D \phi_{T}\left(r_{\nu}\right)^{-1} / \alpha_{T}$.

Thus, we have derived $R, S, A$ such that $R S A \boldsymbol{\xi}=\mathbf{v}$. Since $\mathbf{v}^{T} D \mathbf{v}=\boldsymbol{\xi}^{T} K \boldsymbol{\xi}$, this means $\boldsymbol{\xi}^{T} A^{T} S^{T} R^{T} D R S A \boldsymbol{\xi}=\boldsymbol{\xi}^{T} K \boldsymbol{\xi}$. Since this holds for all $\boldsymbol{\xi} \in \mathbb{R}^{n}$, and since a symmetric matrix $C$ is uniquely determined by the mapping $\boldsymbol{\xi} \mapsto \boldsymbol{\xi}^{T} C \boldsymbol{\xi}$, this means that $K=A^{T} S^{T} R^{T} D R S A$. 
Let us define $J=R S$. Then $K=A^{T} J^{T} D J A$. This factorization underlies the new preconditioning approach. Our factorization is reminiscent of one proposed by Argyris [1] of the form $K=\tilde{A} P \tilde{A}^{T}$, which he calls the "natural factorization." In Argyris's factorization, however, the matrix $\tilde{A}$ has all +1 and 0 entries and therefore is not a node-arc incidence matrix. The purpose of Argyris's matrix $\tilde{A}$ is to assemble the element stiffness matrices, which constitute the blocks of the block-diagonal matrix $P$.

Observe that $J$, a $d q m \times(l-1) m$ matrix, has the $d q \times(l-1)$ matrix

$$
J_{T}=\operatorname{diag}\left(D \phi_{T}\left(r_{1}\right)^{-1}, \ldots, D \phi_{T}\left(r_{q}\right)^{-1}\right) S_{\mathcal{Q}, p} / \alpha_{T}
$$

in columns and rows indexed by $T$, and is zero in all other positions.

6. Analysis of the factorization. In this section, we provide estimates for the norm and condition number of $J$ and of the individual blocks of $D$.

We start by defining some helpful scalars:

$$
\begin{aligned}
\alpha_{T} & =\max _{z \in T_{0}}\left\|D \phi_{T}(z)^{-1}\right\|, \\
\beta_{T} & =\max _{z \in T_{0}}\left\|D \phi_{T}(z)\right\|, \\
\kappa(\mathcal{T}) & =\max _{T \in \mathcal{T}} \alpha_{T} \cdot \beta_{T} .
\end{aligned}
$$

Recall that $\alpha_{T}$ is used in the factorization described herein, which means that $\alpha_{T}$ must be computed for each $T$. In the case $p=1$ (linear elements), finding $\alpha_{T}$ is quite standard. For higher-order elements, there is no simple method to compute this quantity, but an upper bound can be derived using the techniques of [14]. It turns out, however, that an alternative definition

$$
\alpha_{T}=\max _{\nu=1, \ldots, q}\left\|D \phi_{T}\left(r_{\nu}\right)^{-1}\right\|
$$

is also valid for the analysis that follows, and this definition is much simpler to evaluate. The definition of $\beta_{T}$ can be similarly altered. Finally, it should be noted that $\kappa(\mathcal{T}) \geq 1$ since for any $z,\left\|D \phi_{T}(z)^{-1}\right\| \cdot\left\|D \phi_{T}(z)\right\| \geq 1$. It will be seen shortly that the condition numbers of both $J$ and $\bar{J}$ depend on $\kappa(\mathcal{T})$. Therefore, we tacitly assume that $\kappa(\mathcal{T})$ is not too large. This is equivalent to requiring that all the elements in the mesh are "well-shaped", i.e., they are not too distorted when compared to the reference element. This assumption does not imply that the elements are of a uniform size: a uniform resizing of an element $T$ does not affect the product $\alpha_{T} \cdot \beta_{T}$. Note also by Hadamard's inequality that

$$
\alpha_{T}^{-d} \leq \operatorname{det}\left(D \phi_{T}(z)\right) \leq \beta_{T}^{d}
$$

Note that $\alpha_{T}^{-d}$ and $\beta_{T}^{d}$ differ by at most a factor $\kappa(\mathcal{T})^{d}$.

We use these scalars to bound the condition numbers of the factors in our factorization. Let

$$
\sigma_{\mathcal{Q}, p}=\sigma_{\max }\left(S_{\mathcal{Q}, p}\right)
$$

and

$$
\tau_{\mathcal{Q}, p}=\sigma_{\min }\left(S_{\mathcal{Q}, p}\right)
$$


Recall by Lemma 5.1 that both of these are positive.

Lemma 6.1. Matrix $J$ is well-conditioned in the sense that its singular values are bounded between the following values:

$$
\sigma_{\max }(J) \leq \sigma_{\mathcal{Q}, p}
$$

and

$$
\sigma_{\min }(J) \geq \tau_{\mathcal{Q}, p} / \kappa(\mathcal{T})
$$

Proof. Since $J$ is block-diagonal, its maximum singular value is the maximum singular value among any of its blocks and similarly for its minimum singular value. Let $R_{T}$ be the $d q \times d q$ submatrix of $R$ associated with element $T$. Then $J_{T}=R_{T} S_{\mathcal{Q}, p}$. Since in general $\sigma_{\max }(A B) \leq \sigma_{\max }(A) \sigma_{\max }(B)$,

$$
\begin{aligned}
\sigma_{\max }\left(J_{T}\right) & \leq \sigma_{\max }\left(R_{T}\right) \sigma_{\max }\left(S_{\mathcal{Q}, p}\right) \\
& =\sigma_{\max }\left(\operatorname{diag}\left(D \phi_{T}\left(r_{1}\right)^{-1}, \ldots, D \phi_{T}\left(r_{q}\right)^{-1}\right)\right) \sigma_{\mathcal{Q}, p} / \alpha_{T} \\
& =\max _{\nu=1, \ldots, q} \sigma_{\max }\left(D \phi_{T}\left(r_{\nu}\right)^{-1}\right) \sigma_{\mathcal{Q}, p} / \alpha_{T} \\
& \leq \sigma_{\mathcal{Q}, p}
\end{aligned}
$$

Since $\sigma_{\min }(A B) \geq \sigma_{\min }(A) \sigma_{\min }(B)$ for two matrices $A, B$ with full column rank,

$$
\begin{aligned}
\sigma_{\min }\left(J_{T}\right) & \geq \sigma_{\min }\left(R_{T}\right) \sigma_{\min }\left(S_{T}\right) \\
& =\sigma_{\min }\left(\operatorname{diag}\left(D \phi_{T}\left(r_{1}\right)^{-1}, \ldots, D \phi_{T}\left(r_{q}\right)^{-1}\right)\right) \tau_{\mathcal{Q}, p} / \alpha_{T} \\
& =\min _{\nu=1, \ldots, q} \sigma_{\min }\left(D \phi_{T}\left(r_{\nu}\right)^{-1}\right) \tau_{\mathcal{Q}, p} / \alpha_{T} \\
& =\min _{\nu=1, \ldots, q}\left(1 / \sigma_{\max }\left(D \phi_{T}\left(r_{\nu}\right)\right) \tau_{\mathcal{Q}, p} / \alpha_{T}\right. \\
& \geq\left(1 / \beta_{T}\right) \cdot \tau_{\mathcal{Q}, p} / \alpha_{T} \\
& \geq \tau_{\mathcal{Q}, p} / \kappa(\mathcal{T})
\end{aligned}
$$

Recall that the entries of $D$ associated with element $T$ consist of the $d q \times d q$ matrix

$$
D_{T}=\alpha_{T}^{2} \operatorname{diag}\left(\theta\left(\phi_{T}\left(r_{1}\right)\right) \operatorname{det}\left(D \phi_{T}\left(r_{1}\right)\right) \omega_{1} I, \ldots, \theta\left(\phi_{T}\left(r_{q}\right)\right) \operatorname{det}\left(D \phi_{T}\left(r_{q}\right)\right) \omega_{q} I\right)
$$

where $I$ is the $d \times d$ identity matrix. We now define

$$
\hat{\theta}=\max _{T}\left(\max _{x \in \operatorname{interior}(T)} \theta(x) / \min _{x \in \operatorname{interior}(T)} \theta(x)\right) .
$$

We assume that $\hat{\theta}$ is a modest constant. This assumption is realistic because if $\theta$ has steep gradients in a portion of $\tilde{\Omega}$, then small elements are required in that portion of the domain for finite element accuracy. Note that $\theta$ can have discontinuous jumps of arbitrarily large magnitude and yet $\hat{\theta}$ will still be modest provided that the large jumps occur along boundaries of mesh elements (so that the max and min within any particular element do not involve the discontinuity). As before, it suffices to define $\hat{\theta}$ as the maximum ratio over only those points $x$ from the list $\phi_{T}\left(r_{1}\right), \ldots, \phi_{T}\left(r_{q}\right)$.

With this assumption, it is not hard to show that $D_{T}$ is a well conditioned diagonal matrix. Because it is well conditioned, it may be commuted with $J_{T}$ to obtain the 
factorization that is ultimately needed for our preconditioner. Let $M_{\mathcal{Q}}$ be defined as $\max _{i=1, \ldots, q} \omega_{i}$ and $m_{\mathcal{Q}}$ as $\min _{i=1, \ldots, q} \omega_{i}$. Clearly these constants depend only on the quadrature scheme. We introduce an $(l-1) m \times(l-1) m$ diagonal matrix $\bar{D}$ and write $K=A^{T} \bar{D}^{1 / 2} \bar{J}^{T} \bar{J} \bar{D}^{1 / 2} A$ where $\bar{J}$ is chosen so that $J^{T} D J=\bar{D}^{1 / 2} \bar{J}^{T} \bar{J} \bar{D}^{1 / 2}$. To make this identity hold, we take $\bar{J}=D^{1 / 2} J \bar{D}^{-1 / 2}$.

Now we explain how to define $\bar{D}$. It is the diagonal matrix with $m$ diagonal blocks, one for each element, such that $\bar{D}_{T}$ (the submatrix associated with element $T)$ is $\theta\left(\phi_{T}\left(r_{1}\right)\right) \operatorname{det}\left(D \phi_{T}\left(r_{1}\right)\right) \alpha_{T}^{2} I$, where $r_{1}$ is the first quadrature point and $I$ denotes the $(l-1) \times(l-1)$ identity matrix.

We have the following analog of Lemma 6.1.

LEMMA 6.2.

$$
\sigma_{\max }(\bar{J}) \leq \hat{\theta}^{1 / 2} \kappa(\mathcal{T})^{d / 2} M_{\mathcal{Q}}^{1 / 2} \sigma_{\mathcal{Q}, p}
$$

and

$$
\sigma_{\min }(\bar{J}) \geq \hat{\theta}^{-1 / 2} \kappa(\mathcal{T})^{-d / 2-1} m_{\mathcal{Q}}^{1 / 2} \tau_{\mathcal{Q}, p}
$$

Proof. Let the diagonal block of $\bar{J}$ associated with $T$ be denoted $\bar{J}_{T}$. Observe that

$$
\begin{aligned}
\bar{J}_{T} & =D_{T}^{1 / 2} R_{T} S_{\mathcal{Q}, p} \bar{D}_{T}^{-1 / 2} \\
& =\theta\left(\phi_{T}\left(r_{1}\right)\right)^{-1 / 2} \operatorname{det}\left(D \phi_{T}\left(r_{1}\right)\right)^{-1 / 2} \alpha_{T}^{-1} D_{T}^{1 / 2} R_{T} S_{\mathcal{Q}, p} \\
& =\hat{D}_{T} R_{T} S_{\mathcal{Q}, p} .
\end{aligned}
$$

where $\hat{D}_{T}=\theta\left(\phi_{T}\left(r_{1}\right)\right)^{-1 / 2} \operatorname{det}\left(D \phi_{T}\left(r_{1}\right)\right)^{-1 / 2} \alpha_{T}^{-1} D_{T}^{1 / 2}$. Let us write out a formula for the entries of this diagonal matrix:

$$
\begin{aligned}
\hat{D}_{T}= & \theta\left(\phi_{T}\left(r_{1}\right)\right)^{-1 / 2} \operatorname{det}\left(D \phi_{T}\left(r_{1}\right)\right)^{-1 / 2} \alpha_{T}^{-1} D_{T}^{1 / 2} \\
= & \theta\left(\phi_{T}\left(r_{1}\right)\right)^{-1 / 2} \operatorname{det}\left(D \phi_{T}\left(r_{1}\right)\right)^{-1 / 2} \\
& \quad \operatorname{diag}\left(\theta\left(\phi_{T}\left(r_{1}\right)\right) \operatorname{det}\left(D \phi_{T}\left(r_{1}\right)\right) \omega_{1} I, \ldots, \theta\left(\phi_{T}\left(r_{q}\right)\right) \operatorname{det}\left(D \phi_{T}\left(r_{q}\right)\right) \omega_{q} I\right)^{1 / 2} \\
= & \operatorname{diag}\left(\frac{\theta\left(\phi_{T}\left(r_{1}\right)\right) \operatorname{det}\left(D \phi_{T}\left(r_{1}\right)\right) \omega_{1} I}{\theta\left(\phi_{T}\left(r_{1}\right)\right) \operatorname{det}\left(D \phi_{T}\left(r_{1}\right)\right)}, \ldots, \frac{\theta\left(\phi_{T}\left(r_{q}\right)\right) \operatorname{det}\left(D \phi_{T}\left(r_{q}\right)\right) \omega_{q} I}{\theta\left(\phi_{T}\left(r_{1}\right)\right) \operatorname{det}\left(D \phi_{T}\left(r_{1}\right)\right)}\right)^{1 / 2}
\end{aligned}
$$

Recalling the definition of $\hat{\theta}$ as well as (6.5), this formula makes it apparent that

$$
\sigma_{\max }\left(\hat{D}_{T}\right) \leq\left(\hat{\theta} \kappa(\mathcal{T}) M_{\mathcal{Q}}\right)^{1 / 2}
$$

and

$$
\sigma_{\min }\left(\hat{D}_{T}\right) \geq \hat{\theta}^{-1 / 2} \kappa(\mathcal{T})^{-d / 2} m_{\mathcal{Q}}^{1 / 2}
$$

Use the bounds on singular values of $R_{T}$ and $S_{\mathcal{Q}, p}$ derived in the proof of Lemma 6.1 to conclude the theorem. 
7. The piecewise linear two-dimensional case. In this section, we specialize the theory developed so far to the case that $p=1, d=2, q=1, r_{1}=(1 / 3,1 / 3)$, $\omega_{1}=1 / 2$, that is, the case of piecewise linear two-dimensional triangular elements integrated with midpoint quadrature, which is a common case in practice. The notation is simplified, and several other simplifications to the analysis are possible.

Midpoint quadrature is accurate for constant and linear functions, and in particular, accurate up to degree $2 p-2$ so the two assumptions in Theorem 3.1 are valid for this schema.

In this case, each $\phi_{T}$ is an affine linear function, $\tilde{\Omega}$ is a polygon, and $\tilde{\Gamma}_{1}$ is a piecewise-linear path. We have $l=3$, the three nodes are $z_{1}=(0,0), z_{2}=(1,0)$, $z_{3}=(0,1)$, and the three affine linear shape functions are $N_{1}(\xi, \eta)=1-\xi-\eta$, $N_{2}(\xi, \eta)=\xi, N_{3}(\xi, \eta)=\eta$.

In the factorization of $K=A^{T} S^{T} R^{T} D R S A, D$ is a $2 m \times 2 m$ diagonal matrix whose diagonal entries are of the form $\theta_{T} \operatorname{det}\left(D \phi_{T}\right) \alpha_{T}^{2}$, where $\theta_{T}$ is the value of $\theta\left(\phi_{T}(1 / 3,1 / 3)\right)$, i.e., the value of $\theta$ at the mapped centroid of the element, $\operatorname{det}\left(D \phi_{T}\right)$ is the determinant of the $2 \times 2$ matrix associated with mapping element $T$ (which is constant over the element), and $\alpha_{T}=\sigma_{\min }\left(D \phi_{T}\right)$.

The matrix $S_{\mathcal{Q}, p}$ in this case turns out to be $I(2 \times 2$ identity matrix $)$ since the two finite differences $U_{T}\left(z_{2}\right)-U_{T}\left(z_{1}\right)$ and $U_{T}\left(z_{3}\right)-U_{T}\left(z_{1}\right)$ are exactly the two entries of $\nabla U_{T}$ (which is constant over $T_{0}$ ). Therefore, $\sigma_{\mathcal{Q}, p}=\tau_{\mathcal{Q}, p}=1$. The matrix $R_{T}$ is also $2 \times 2$ and is $D \phi_{T}^{-1} / \alpha_{T}$. Thus, $J_{T}=D \phi_{T}^{-1} / \alpha_{T}$.

For each element, $\alpha_{T}=\left\|D \phi_{T}^{-1}\right\|$ and $\beta_{T}=\left\|D \phi_{T}\right\|$. Thus, $\alpha_{T} \beta_{T}=\kappa_{2}\left(D \phi_{T}\right)$. It can be shown that this quantity, called the aspect ratio of $T$, is within a constant factor of the reciprocal of the minimum angle of $T$. Thus, $\kappa(\mathcal{T})$ is a modest constant provided no triangle in the mesh has a very sharp angle.

Specializing Lemma 6.1 , we find that $\sigma_{\min }(J) \geq 1 / \kappa(\mathcal{T})$ and $\sigma_{\max }(J) \leq 1$. The constant $\hat{\theta}$ for this case is identically 1 regardless of the function $\theta(x)$ since, as noted above, it is the ratio of $\theta$ evaluated at two Gauss points of an element, but each element has only one Gauss point. Similarly, the factor $\kappa(\mathcal{T})^{d / 2}$ in Lemma 6.2 goes away because it arises as a ratio of max versus min determinants in an element, but the only point where the determinant is evaluated is at the centroid so the ratio is always 1. Therefore, Lemma 6.2 simplifies to $\sigma_{\max }(\bar{J}) \leq 1 / 2$ and $\sigma_{\min }(\bar{J}) \geq 1 /(2 \kappa(\mathcal{T}))$.

8. Preconditioning Strategy Summary. In this section we summarize how our theory can be used to construct provably good preconditioners for finite element systems. As mentioned earlier, we know from the literature how to precondition Laplacian-type systems of the form $A^{T} D A$ where $A$ is a node-arc incidence matrix. We have a factorization $K=A^{T} J D J^{T} A$, but the standard theory does not apply because of the presence of $J$. So we refactor as in Lemma 6.2 to obtain $K=A^{T} \bar{D}^{1 / 2} \bar{J}^{T} \bar{J} \bar{D}^{1 / 2} A$. Now we can apply Theorem 2.4 by taking

$$
V=A^{T} \bar{D}^{1 / 2}, G=\bar{J}^{T},
$$

and get

$$
\kappa(K, \bar{K}) \leq \kappa(\bar{J})^{2}
$$

where $\bar{K}=A^{T} \bar{D} A$. This is the first step in our preconditioning scheme: approximate $K$ by $\bar{K}$.

As an aside, consider the general case of preconditioning $A^{T} J D J^{T} A$ with $D$ diagonal. Ideally, we want to choose $\bar{D}$ such that $\kappa(\bar{J})$ is minimized subject to 
$J^{T} D J=\bar{D}^{1 / 2} \bar{J}^{T} \bar{J} \bar{D}^{1 / 2}$. Finding the optimal scaling $\bar{D}$ is hard, but a good (nearoptimal) choice can be found efficiently by choosing $\bar{D}$ to be the symmetric scaling of $J^{T} D J$ that makes all the diagonal entries one. This implies

$$
\bar{D}_{i i}=\left(J^{T} D J\right)_{i i}, \quad i=1,2, \ldots
$$

On the other hand, there is no guarantee for the general case that the resulting $\bar{J}$ is well conditioned. In the case of the finite element method for (4.1), there is special structure in $J$ and $D$ that allows us to prove a result like Lemma 6.2.

The preconditioning algorithm goes as follows. First compute $\bar{D}$ as given above. Then construct a good preconditioner for $\bar{K}=A^{T} \bar{D} A$. This is easy because many preconditioners work well on symmetric diagonally dominant systems. For example, a near-optimal preconditioner was recently given by Spielman and Teng [13]. We remark that the algorithm of Spielman and Teng is quite complex (and has not been implemented yet), so in practice a better choice might be the augmented max-weight spanning trees first suggested by Vaidya and described in $[2,4]$. In fact, any solver can be used for the symmetric M-matrix $A^{T} \bar{D} A$, including multigrid and AMG methods. This allows us to reuse existing algorithms and software.

The triangle inequality (2.1) allows us to combine the two steps. In particular, if Spielman and Teng's preconditioner $M$ approximates $\bar{K}$ well in the sense that $\kappa(\bar{K}, M)$ is small, and our result above shows that $\kappa(K, \bar{K})$ is also small, then $\kappa(M, K)$ is small.

Our approximation scheme could be rewritten on an element-by-element basis. Consider an element matrix $K_{i}=A_{i}^{T} J_{i}^{T} D_{i} J_{i} A_{i}$, where each row of $A_{i}$ is also a row of $A$ and thus has the special sparsity structure discussed earlier. We find a $\bar{D}_{i}$ that is a good approximation to $J_{i}^{T} D_{i} J_{i}$. Thus, we let $\bar{K}_{i}=A_{i}^{T} \bar{D}_{i} A_{i}$. The overall approximation is $\bar{K}=\sum_{i} \bar{K}_{i}$. Alternatively, we can take a global view as in the above analysis and write $\bar{K}=A^{T} \bar{D} A$, where $A=\left(A_{1} ; A_{2} ; \ldots ; A_{m}\right)$ and $\bar{D}=$ $\operatorname{diag}\left(\bar{D}_{1}, \bar{D}_{2}, \ldots, \bar{D}_{m}\right)$. To simplify notation, we have adopted the global view in this paper, but the reader should keep in mind that our approximation can take place element by element, which may be important in an implementation.

8.1. Total work. Suppose that we have an iterative algorithm that can solve symmetric, diagonally dominant M-matrices in $f(n, m)$ operations, where $n$ is the number of variables and $m$ the number of nonzeros. Spielman and Teng [13] have recently proven that such systems can be solved in near-linear time, that is, $f(n, m)=$ $\tilde{O}(m)$, where $\tilde{O}$ means that some logarithmic factors have been omitted. We have shown that all finite element systems that discretize (4.1) in the usual way can be solved using such an algorithm and the condition number only increases by a factor $\kappa(\bar{J})^{2}$. Thus the number of iterations (and the work) increases by at most $\kappa(\bar{J})$. Consequently, we can solve finite element systems with work $\kappa(\bar{J}) f(n, m)$. When $\kappa(\bar{J})$ is bounded by a constant, the total work is asymptotically the same as for symmetric, diagonally dominant M-matrices, that is, almost linear. This is true even for arbitrarily ill-conditioned systems.

For a given problem instance it is easy to estimate $\kappa(\bar{J})$, since $\bar{J}$ can readily be computed from the given $J$. When $J$ is block diagonal, the extreme eigenvalues of each block can be computed cheaply. One interpretation of our analysis is that the condition number (of the preconditioned system) is proportional to a factor that depends on the quality of the mesh.

9. Open questions. This work is the first to extend support-tree methods, which previously have been shown to be good preconditioners for diagonally dominant 
matrices with negative off-diagonal entries, to the class of finite element matrices. We have shown that the scope of the method includes the standard scalar elliptic boundary value problem, but perhaps the scope of finite element problems that can be tackled with this method could be expanded further.

One generalization would be the class of problems $\nabla \cdot(\Theta(x) \nabla u)=-f$, where $\Theta(x)$ is a spatially varying $d \times d$ symmetric positive definite matrix. This generalization would present problems for our current analysis in the case that $\Theta(x)$ is highly illconditioned. It would still be straightforward to write $K=A^{T} J^{T} D J A$ where $D$ is now block diagonal, but our analysis of the introduction of $\bar{J}$ would run into trouble because the $d q \times d q$ diagonal blocks of $D$ are no longer individually well conditioned.

It would also be interesting to tackle vector problems such as linear elasticity or Stokes' flow, or higher-order equations like the biharmonic equation. It seems likely that our techniques can extend to at least some of these problems since they all have a symmetric positive definite weak form. On the other hand, a recent result [5] suggests that the extension of our results to linear elasticity will not be straightforward because of the higher nullity of element stiffness matrices in the case of linear elasticity. A further generalization would be to unsymmetric problems like the convection-diffusion equation. The latter class of problems would require substantial rethinking of the whole approach since condition number reduction, which is very relevant for the application of conjugate gradient to symmetric positive definite systems, is less relevant to the application of GMRES to unsymmetric systems.

Our analysis is based on condition numbers (support numbers). One drawback of this approach is that the convergence and work estimates may be too pessimistic. For instance, the condition number of the preconditioned linear systems depends on $\kappa(\mathcal{T})$, the worst aspect ratio of any element in the mesh. If there is only one poorly shaped element in the mesh, we expect iterative solvers will only take a few extra iterations since changing a single element implies a low-rank correction to the assembled stiffness matrix. Any analysis based on condition numbers will be unable to capture this effect. A related open issue is whether we can exploit recent work in mesh quality metrics [11] to show that "good meshes" both have small error in the FEM approximation and also produce linear systems that can be well approximated by diagonally dominant systems.

Another point to make about our method is that, although the condition number of the preconditioned system has an upper bound independent of

$$
R_{\theta}=\max _{x \in \Omega} \theta(x) / \min _{x \in \Omega} \theta(x),
$$

there will still be a loss of significant digits due to roundoff error when using our method in the case that $R_{\theta}$ is large. This is because the system matrix and the preconditioner separately are ill-conditioned operators. A special case of (4.1) in which $R_{\theta}$ is extremely large and in which $f=0$ was considered in [15]. That paper proposed a method based on Gaussian elimination in which the loss of significant digits is avoided. Some of the ideas behind [15] were also extended to solution via conjugate gradient using support preconditioners by [9]. The methodology in [9], however, was for node-arc adjacency matrices and for a particular kind of support preconditioner called a support tree [7], and it is not clear whether that method would apply to the present setting.

Acknowledgments. The work of the first two authors was funded by the Applied Mathematical Sciences program, U.S. Department of Energy, Office of Energy 
Research and performed at Sandia National Labs, a multiprogram laboratory operated by Sandia Corporation, a Lockheed Martin Company, for the U.S. Dept. of Energy's National Nuclear Security Administration under contract number DE-AC94AL85000. The third author was supported in part by NSF Grant CCF-0085969.

\section{REFERENCES}

[1] J. H. Argyris. The natural factor formulation of the stiffness for the matrix displacement method. Computer Methods in Applied Mechanics and Engineering, 5:97-119, 1975.

[2] M. Bern, J. R. Gilbert, B. Hendrickson, N. Nguyen, and S. Toledo. Support-graph preconditioners. Technical report, School of Computer Science, Tel-Aviv University, 2001. Submitted for publication. A shorter version appeared in Proceedings of the 5th Copper Mountain Conference On Iterative Methods, 1998.

[3] E. G. Boman and B. Hendrickson. Support theory for preconditioning. SIAM J. on Matrix Anal. and Appl., 25(3):694-717, 2004. (Published electronically on 17 Dec 2003.).

[4] D. Chen and S. Toledo. Vaidya's preconditioners: Implementation and experimental study. ETNA, 16, 2003. Available from http://etna.mcs.kent.edu.

[5] D. Chen and S. Toledo. Combinatorial characterization of the null spaces of symmetric Hmatrices. To appear in Linear Algebra and its Applications, 2004.

[6] R. Cools. An encyclopaedia of cubature formulas. J. Complexity, 19:445-453, 2003.

[7] K. Gremban. Combinatorial Preconditioners for Sparse, Symmetric, Diagonally Dominant Linear Systems. PhD thesis, School of Computer Science, Carnegie-Mellon University, 1996. Available as Tech. Report CMU-CS-96-123.

[8] I. Gustafsson. An incomplete factorization preconditioning method based on modification of element matrices. BIT, 36:86-100, 1996.

[9] V. E. Howle and S. A. Vavasis. An iterative method for solving complex-symmetric systems arising in electrical power modeling. Submitted to SIAM J. Matrix Analysis App., 2002.

[10] C. Johnson. Numerical Solution of Partial Differential Equations by the Finite Element Method. Cambridge University Press, 1987.

[11] P. Knupp. Algebraic mesh quality metrics. SIAM J. Sci. Comput., 23:193-218, 2001.

[12] M. Lenoir. Optimal isoparametric finite elements and error estimates for domains involving curved boundaries. SIAM J. Numer. Anal., 23:562-580, 1986.

[13] D. Spielman and S-H. Teng. Nearly-linear time algorithms for graph partitioning, graph sparsification, and solving linear systems. Manuscript available from www.arxiv.org/abs/cs.DS/0310051, 2003.

[14] S. Vavasis. A Bernstein-Bézier sufficient condition for invertibility of polynomial mappings. Archived by http://arxiv.org/abs/cs.NA/0308021, 2003.

[15] S. A. Vavasis. Stable finite elements for problems with wild coefficients. SIAM J. Numer. Anal., 33:890-916, 1996. 